\title{
Rocky super-Earth interiors
}

\section{Structure and internal dynamics of CoRoT-7b and Kepler-10b}

\author{
F. W. Wagner ${ }^{1,2}$, N. Tosi ${ }^{1,3}$, F. Sohl ${ }^{1}$, H. Rauer ${ }^{1,4}$, and T. Spohn ${ }^{1,2}$ \\ ${ }^{1}$ Institute of Planetary Research, German Aerospace Center (DLR), Rutherfordstrasse 2, 12489 Berlin-Adlershof, Germany \\ ${ }^{2}$ Institute for Planetology, Westphalian Wilhelms-University (WWU), Wilhelm-Klemm-Strasse 10, 48149 Münster, Germany \\ 3 Department of Geodesy, Technical University of Berlin (TUB), Strasse des 17. Juni 135, 10623 Berlin, Germany \\ ${ }^{4}$ Center of Astronomy and Astrophysics, Technical University of Berlin (TUB), Hardenbergstrasse 36, 10623 Berlin, Germany
}

Received 11 November 2011 / Accepted 28 February 2012

\begin{abstract}
Aims. We present interior structure models of the recently discovered exoplanets CoRoT-7b and Kepler-10b addressing their bulk compositions, present thermal states, and internal dynamics. We investigate how mantle convection patterns are influenced by the depth-dependence of thermodynamic parameters (e.g., thermal expansivity and conductivity) caused by the extended pressure and temperature ranges within rocky super-Earths.

Methods. To model the interior of rocky exoplanets, we construct a four-layer structural model solving the mass and energy balance equations in conjunction with a generalized Rydberg equation of state providing the radial density distribution within each layer. The present thermal state is calculated according to a modified mixing-length approach for highly viscous fluids. Furthermore, the obtained internal structure is used to carry out two-dimensional convection simulations to visualize the mantle convection pattern within massive exoplanets such as CoRoT-7b and Kepler-10b.

Results. Both CoRoT-7b and Kepler-10b most likely have large iron cores and a bulk composition similar to that of Mercury. For a planetary radius of $R_{\mathrm{p}}=(1.58 \pm 0.10) R_{\oplus}$, a revised total mass of $M_{\mathrm{p}}=(7.42 \pm 1.21) M_{\oplus}$, and the existence of a third planet in the CoRoT-7 planetary system, calculations suggest that an iron core of $64 \mathrm{wt}-\%$ and a silicate mantle of $36 \mathrm{wt}-\%$ is produced owing to the relatively high average compressed density of $\rho_{\text {avg }}=(10.4 \pm 1.8) \mathrm{g} \mathrm{cm}^{-3}$. Kepler-10b's planetary radius and total mass yield an iron core of $59.5 \mathrm{wt}-\%$, which complements the silicate mantle of $40.5 \mathrm{wt}-\%$. An enhanced radiogenic heating rate owing to CoRoT-7b's young age (1.2-2.3 Gyr) raises the radial distribution of temperature by only a few hundred Kelvin, but reduces the viscosity by an order of magnitude. The planform of mantle convection is found to be strongly modified for depth-dependent material properties, with hot plumes rising across the whole mantle and cold slabs, which stagnate in the mid-mantle because of the loss of buoyancy.

Conclusions. We use a new model approach to determine the detailed interior structures and present thermal states of CoRoT-7b and Kepler-10b. Both planets are found to be enriched in iron. The results imply that modest radiogenic heating does not play a significant role in determining the internal structure of rocky exoplanets. The depth-dependence of thermodynamic properties, however, strongly influences the mantle convection patterns within exoplanets such as CoRoT-7b and Kepler-10b. This may have a significant effect on the thermal evolution and magnetic field generation of close-in super-Earths.
\end{abstract}

Key words. planets and satellites: interiors - planets and satellites: composition - planets and satellites: individual: CoRoT-7b planets and satellites: individual: Kepler-10b

\section{Introduction}

CoRoT-7b was discovered by the CoRoT space telescope in 2009. It represents the first exoplanet among more than 30 lowmass $\left(<15 M_{\oplus}\right)$ extrasolar planets for which the planetary radius and total mass have been measured. In units of Earth equivalent quantities, these are $R_{\mathrm{p}}=(1.58 \pm 0.10) R_{\oplus}$ and $M_{\mathrm{p}}=(5.2 \pm$ 0.8) $M_{\oplus}$ (Bruntt et al. 2010), respectively. These values yield an average density for CoRoT-7b of $\rho_{\text {avg }}=(7.2 \pm 1.8) \mathrm{g} \mathrm{cm}^{-3}$, which is comparable to the Earth's density $\left(5.515 \mathrm{~g} \mathrm{~cm}^{-3}\right)$ and indicative of a terrestrial bulk composition. However, a reanalysis of radial velocity data by Hatzes et al. (2010) found evidence of a third planet (CoRoT-7d), which implies that CoRoT-7b has a higher planetary mass of $M_{\mathrm{p}}=(7.42 \pm 1.21) M_{\oplus}$ and an average density of $\rho_{\text {avg }}=(10.4 \pm 1.8) \mathrm{g} \mathrm{cm}^{-3}$ (Hatzes et al. 2011). The orbital parameters of CoRoT-7b are extreme. With a semimajor axis of $a=(0.0172 \pm 0.0003) \mathrm{AU}$, the planet orbits its G-type parent star CoRoT-7 within a period of $P=0.854$ days.
In comparison to the Sun, CoRoT-7 has a slightly higher metallicity $[\mathrm{Fe} / \mathrm{H}]=+0.03 \pm 0.06$ and a slightly lower effective surface temperature of $T_{\star}=(5250 \pm 60) \mathrm{K}$. The age of the star and its planetary system, which harbors at least another low-mass exoplanet, CoRoT-7c, is expected to be 1.2-2.3 Gyr.

The Kepler mission announced the discovery of a comparable transiting exoplanet, Kepler-10b, with a planetary radius of $R_{\mathrm{p}}=\left(1.416_{-0.036}^{+0.033}\right) R_{\oplus}$ and a total mass of $M_{\mathrm{p}}=$ $\left(4.56_{-1.29}^{+1.17}\right) M_{\oplus}$ (Batalha et al. 2011). This yields a relatively high average density of $\rho_{\text {avg }}=\left(8.8_{-2.9}^{+2.1}\right) \mathrm{g} \mathrm{cm}^{-3}$ compared to those of both the Earth and CoRoT-7b. The planet orbits Kepler-10 in only $P=\left(0.837495_{-0.000005}^{+0.00004}\right)$ days at a close distance of $a=$ $\left(0.01684_{-0.00034}^{+0.00032}\right)$ AU. The G-type parent star is slightly depleted in metals according to its metallicity of $[\mathrm{Fe} / \mathrm{H}]=-0.15 \pm 0.04$ and has an effective surface temperature of $T_{\star}=(5627 \pm 44) \mathrm{K}$. In contrast to the relatively young planetary system of CoRoT-7, Kepler-10 is an old star of $(11.9 \pm 4.5)$ Gyr. 
Table 1. Model input parameters as obtained from observations or based on reasonable assumptions.

\begin{tabular}{lccccc}
\hline \hline & $\mathrm{C} 1$ & $\mathrm{C} 2$ & $\mathrm{C} 3$ & $\mathrm{~K} 1$ & Earth \\
\hline$M_{\mathrm{p}}\left[10^{24} \mathrm{~kg}\right]$ & 31.063 & 31.063 & 44.324 & 27.240 & $5.9736^{a}$ \\
$R_{\mathrm{p}}[\mathrm{km}]$ & 10070 & 10070 & 10070 & 9021 & $6371^{a}$ \\
$\rho_{\mathrm{avg}}\left[\mathrm{g} \mathrm{cm}^{-3}\right]$ & 7.2 & 7.2 & 10 & 8.8 & $5.515^{a}$ \\
$T_{\mathrm{s}}[\mathrm{K}]$ & 1300 & 1300 & 1300 & 1300 & $300^{b}$ \\
$\epsilon_{\mathrm{m}}\left[\mathrm{pW} \mathrm{kg}{ }^{-1}\right]$ & 7.39 & 36.9 & 7.39 & 7.39 & $7.39^{c}$ \\
$p_{\mathrm{s}}[\mathrm{hPa}]$ & 0. & 0. & 0. & 0. & $1010^{d}$ \\
\hline
\end{tabular}

Notes. Values for the Earth are given for comparison.

References. ${ }^{(a)}$ Romanowicz \& Lambeck (1977); ${ }^{(b)}$ Stacey \& Davis (2008); ${ }^{(c)}$ Turcotte \& Schubert (2002); ${ }^{(d)}$ Dziewonski \& Anderson (1981).

In the present study, we apply these observational constraints to construct interior structure models for CoRoT-7b and Kepler$10 \mathrm{~b}$ and infer their bulk compositions and present thermal states. We begin in Sect. 2 with a brief description of how we calculate the radial structure and depth-dependent thermodynamic quantities for CoRoT-7b and Kepler-10b. In the following Sect. 3, we present our findings and possible implications for the interior structure, thermal state, and bulk composition of CoRoT-7b and Kepler-10b. Finally, in Sect. 4, the results and their effects on the interior dynamics (e.g., mantle convection pattern) for both planets are discussed and some conclusions are drawn.

\section{Modeling approach}

The following models have been considered for CoRoT-7b: (a) model $\mathrm{C} 1$ represents a terrestrial planet with a total mass of 5.2 $M_{\oplus}$ and a specific mantle heat production rate similar to the present-day value of the Earth, (b) model C2 is identical to $\mathrm{C} 1$ with the exception of a five times higher specific heat production rate in the planetary mantle relative to the present-day value of the Earth, and (c) model C3 represents an iron-enriched planet with a total mass of $7.42 M_{\oplus}$ and a specific mantle heat production rate similar to the present-day value of the Earth. All three CoRoT-7b cases have the same planetary radius of $1.58 R_{\oplus}$. For Kepler-10b, only one case (K1) has been considered for which we use the observed values of planetary radius, total mass, and an Earth-like value for the radiogenic heating. All model input parameters are listed in Table 1.

Owing to the planet's close proximity to its primary, extreme surface temperatures $T_{\mathrm{s}}$ are expected. The possible range of surface temperatures for CoRoT-7b and Kepler-10b can be estimated using the following approach (e.g., Barnes et al. 2010). If we assume the planetary surface to be uniformly heated,

$T_{\mathrm{s}}=(1-A)^{1 / 4} \tau\left(\frac{R_{\star}}{2 a}\right)^{1 / 2} T_{\star}$.

This equation assumes an efficient heat transport throughout the planetary surface. If we instead neglect any heat transfer from the day side to the night side of the planet,

$T_{\mathrm{S}}=(1-A)^{1 / 4} \tau\left(\frac{R_{\star}}{a}\right)^{1 / 2} T_{\star}$,

where $A$ is the albedo, $\tau$ quantifies the effectiveness of heat transport, $a$ is the distance of the planet to its host star, and $R_{\star}$ and
$T_{\star}$ are the radius and the effective temperature of the star, respectively. Assuming zero albedo and no greenhouse effect, the effective equilibrium temperature at the planet's sub-stellar point is $T_{\mathrm{s}}=(2560 \pm 125) \mathrm{K}$ according to Eq. (2). Assuming an efficient heat transport mechanism to distribute the energy uniformly throughout the planet's surface, the temperature would be about $T_{\mathrm{s}}=1810 \mathrm{~K}$ according to Eq. (1). If we were to assume a Bond albedo similar to that of Venus, the surface temperature would still be as high as $1270 \mathrm{~K}$. Although Venus' albedo of $\sim 0.8$ represents an extreme case for terrestrial bodies in the Solar System, a similarly high albedo of $0.5 \pm 0.4$ has been suggested for Kepler-10b owing to back-scattering from small solid particles of non-absorbing refractory compounds such as $\mathrm{Al}_{2} \mathrm{O}_{3}$ floating on top of extended regions of molten rock (Rouan et al. 2011).

As a consequence of the planet's intense heating due to stellar irradiation, a high rate of volatile evaporation is expected (Valencia et al. 2010). Hence, volatiles should be effectively degassed from the planetary interior so that it is most likely that CoRoT-7b and Kepler-10b are dry exoplanets. Here, we consider the case of fully differentiated interiors with a terrestrial bulk composition. The model planets consist of a silicate mantle, composed primarily of olivine, $\mathrm{MgSiO}_{3}$ perovskite, and $\mathrm{MgSiO}_{3}$ post-perovskite, surrounding an iron core.

\subsection{Mechanical model}

Assuming a spherically symmetric planet in perfect mechanical equilibrium and thermal steady state, its depth-dependent internal structure is described by the coupled differential equations for mass $m(r)$, moment of inertia $\theta(r)$, acceleration of gravity $g(r)$, and pressure $p(r)$ (e.g., Sohl et al. 2009)

$$
\begin{aligned}
& \frac{\mathrm{d} m}{\mathrm{~d} r}=4 \pi r^{2} \rho, \\
& \frac{\mathrm{d} \theta}{\mathrm{d} r}=\frac{8}{3} \pi r^{4} \rho, \\
& \frac{\mathrm{d} g}{\mathrm{~d} r}=4 \pi \mathrm{G} \rho-2 \frac{g}{r}, \\
& \frac{\mathrm{d} p}{\mathrm{~d} r}=-\rho g,
\end{aligned}
$$

where $r$ is the radial distance from the center of the planet, $G$ is the gravitational constant, and $\rho$ is the local density.

The local density is calculated by using a semi-empirical equation of state (EoS). We have chosen to use the isothermal generalized Rydberg EoS (Stacey 2005)

$$
p(\rho)=3 K_{0} x^{K_{\infty}^{\prime}}\left(1-x^{-\frac{1}{3}}\right) \exp \left[\left(\frac{3}{2} K_{0}^{\prime}-3 K_{\infty}^{\prime}+\frac{1}{2}\right)\left(1-x^{-\frac{1}{3}}\right)\right],
$$

where $x=\rho / \rho_{0}$ is the compression ratio with respect to the ambient density $\rho_{0}$. The quantities $K_{0}, K_{0}^{\prime}$, and $K_{\infty}^{\prime}$ are the isothermal bulk modulus and its pressure derivative both at ambient pressure and in the limit of infinitely large pressure, respectively. Table 2 lists the parameters used for the model calculations. The thermal pressure $p_{\text {th }}(\rho, T)$ is calculated using the Mie-Grüneisen-Debye model for the vibrational energy (Jackson \& Rigden 1996)

$p_{\text {th }}(\rho, T)=\frac{\rho^{2}}{\mu}\left(\frac{\mathrm{d} F_{\mathrm{vib}}}{\mathrm{d} \rho}\right)_{T}$, 
Table 2. Fit parameters used for the generalized Rydberg equation of state (EoS).

\begin{tabular}{lcccccccc}
\hline \hline Material (Phase) & $\rho_{0}\left[\mathrm{~g} \mathrm{~cm}^{-3}\right]$ & $K_{0}[\mathrm{GPa}]$ & $K_{0}^{\prime}$ & $K_{\infty}^{\prime}$ & $\gamma_{0}$ & $\gamma_{\infty}$ & $\beta$ & $\lambda$ \\
\hline $\mathrm{Fe}^{a}(\epsilon)$ & 8.2694 & 149.4 & 5.65 & $2.943^{e}$ & 1.875 & 1.305 & 3.289 & - \\
$\mathrm{MgSiO}_{3}{ }^{b}(\mathrm{ppv})$ & 3.9776 & 204.0 & 4.20 & $2.561^{e}$ & $1.553^{f}$ & $1.114^{f}$ & $4.731^{f}$ & - \\
$\mathrm{MgSiO}_{3}{ }^{c}(\mathrm{pv})$ & 4.1059 & 270.6 & 3.81 & $2.62975^{e}$ & $1.506^{f}$ & $1.14821^{f}$ & $7.02469^{f}$ & - \\
$\mathrm{Mg}_{2} \mathrm{SiO}_{4}{ }^{d}(\alpha)$ & 3.2137 & 127.4 & 4.20 & - & 1.31 & - & - & 3.2 \\
\hline
\end{tabular}

Notes. Some values have been refitted to the applied EoS.

References. ${ }^{(a)}$ Dewaele et al. (2006); ${ }^{(b)}$ Oganov \& Ono (2004); ${ }^{(c)}$ Oganov et al. (2001); ${ }^{(d)}$ Katsura et al. $(2009 a) ; ~\left({ }^{(e)}\right.$ fixed according to $K_{\infty}^{\prime}=$ $2\left(\gamma_{\infty}+1 / 6\right) ;{ }^{(f)}$ Ono \& Oganov (2005).

where $F_{\text {vib }}$ is the lattice vibrational part of the Helmholtz free energy and $\mu$ is the molecular mass. The total pressure $p(\rho, T)$ at a given density and temperature can then be expressed as

$p(\rho, T)=p(\rho)+p_{\text {th }}(\rho, T)$.

We numerically solve the differential equations (Eqs. (3)-(6)) using a BDF (backward differentiation formulae) algorithm (Shampine 1994). The algorithm starts radial integration at the center $(r=0)$ using the central boundary conditions of $m(0)=0$, $\theta(0)=0, g(0)=0$, and $p(0)=p_{\mathrm{c}}$, where $p_{\mathrm{c}}$ is a chosen starting estimate for the central pressure. The integration then proceeds outward through each shell consisting of a specified material until the total mass $M_{\mathrm{p}}$ of the planet is attained. If necessary, this process is repeated with an iteratively adjusted central pressure $p_{\mathrm{c}}$. The algorithm stops integrating when the surface boundary conditions of $m\left(R_{\mathrm{p}}\right)=M_{\mathrm{p}}$ and $p\left(R_{\mathrm{p}}\right)=p_{\mathrm{s}}$ are met at $r=R_{\mathrm{p}}$.

\subsection{Thermal model}

To calculate self-consistently the radial distribution of temperature within planetary mantles, we adopt the concept of mixing length theory (MLT). The MLT (e.g., Hansen et al. 2004) is commonly used to model the heat transport within stellar interiors. Sasaki \& Nakazawa (1986) reformulated the approach for highly viscous fluids governing low Reynolds number convection in solid planets. This new formulation of the MLT was derived assuming that the Stokes' viscous drag is balanced by the buoyancy force acting on a fluid parcel. Tachinami et al. (2011) demonstrated that the mixing length approach yields reliable results when compared to conventional boundary layer theory.

Starting with the assumption of thermal equilibrium, the total heat flux $q$ through a spherical shell is given as the sum of the conductive and convective heat flux

$q=q_{\mathrm{cond}}+q_{\mathrm{conv}}=-k_{\mathrm{c}} \frac{\mathrm{d} T}{\mathrm{~d} r}+q_{\mathrm{conv}}$,

where $k_{\mathrm{c}}$ is the thermal conductivity and $\mathrm{d} T / \mathrm{d} r$ is the local temperature gradient. According to MLT, we can express the convective heat flux $q_{\text {conv }}$ in Eq. (10) in terms of a heat transfer coefficient $k_{v}$

$q_{\mathrm{conv}}=k_{v}\left[\left(\frac{\mathrm{d} T}{\mathrm{~d} r}\right)_{S}-\frac{\mathrm{d} T}{\mathrm{~d} r}\right]$,

where $(\mathrm{d} T / \mathrm{d} r)_{S}$ and $\mathrm{d} T / \mathrm{d} r$ denote the local adiabatic temperature gradient and the local temperature gradient, respectively. The coefficient $k_{v}$ is given as a function of local parameters only (e.g., Senshu et al. 2002) and can be expressed as

$k_{v}= \begin{cases}0 & \text { for }\left|\frac{\mathrm{d} T}{\mathrm{~d} r}\right|<\left|\left(\frac{\mathrm{d} T}{\mathrm{~d} r}\right)_{S}\right| \\ \frac{l^{4} \alpha^{2} \rho g K_{S}}{18 \gamma \eta_{\mathrm{eff}}}\left[\left(\frac{\mathrm{d} T}{\mathrm{~d} r}\right)_{S}-\frac{\mathrm{d} T}{\mathrm{~d} r}\right] & \left.\text { for }\left|\frac{\mathrm{d} T}{\mathrm{~d} r}\right|>\mid \frac{\mathrm{d} T}{\mathrm{~d} r}\right)_{S} \mid\end{cases}$
Table 3. Creep parameters for a dry olivine rheology used in the determination of viscosity.

\begin{tabular}{lccccc}
\hline \hline Creep & $\begin{array}{c}B \\
{\left[\mathrm{~Pa}^{-n} \mathrm{~s}^{-1} \mathrm{~m}^{m}\right]}\end{array}$ & $m$ & $n$ & $\begin{array}{c}E^{*} \\
{\left[\mathrm{~kJ} \mathrm{~mol}^{-1}\right]}\end{array}$ & $\begin{array}{c}V^{*} \\
{\left[\mathrm{~cm}^{3} \mathrm{~mol}^{-1}\right]}\end{array}$ \\
\hline DF & $6.1 \times 10^{-19}$ & 2.5 & 1.0 & 300 & 6 \\
DL & $2.4 \times 10^{-16}$ & 0. & 3.5 & 540 & 20 \\
\hline
\end{tabular}

Notes. DF: diffusion; DL: dislocation.

References. Karato \& Wu (1993).

where $l$ is the characteristic mixing length, $\alpha$ is the thermal expansivity, $\gamma$ is the thermodynamic Grüneisen parameter, $\eta_{\mathrm{eff}}$ is the effective viscosity, and $K_{S}$ is the adiabatic bulk modulus, which can be obtained by evaluating the equation of state. Following Abe (1997), we treat the mixing length $l$ as the distance from the nearest boundary of the convective layer. Depth variations of the Grüneisen parameter are calculated by applying the Al'tshuler formulation $\gamma=\gamma_{\infty}+\left(\gamma_{0}-\gamma_{\infty}\right) x^{-\beta}$ to the lower mantle and the core (Al'tshuler et al. 1987). For the upper mantle, we use $\gamma=\gamma_{0} x^{-\lambda}$. Table 2 lists the parameters, which are used for the model calculations.

The heat transfer coefficient $k_{v}$ depends on the local viscosity $\eta_{\text {eff }}$ given in Eq. (12). Owing to the high temperature and large pressure within massive terrestrial planets, the viscosity is expected to depend strongly on both temperature and pressure. Hence, we use a temperature- and pressure-dependent Arrhenius-type flow law between the applied shear stresses $\sigma$ and the resulting shear strain rate $\dot{\epsilon}$ (e.g., Ranalli 2001)

$\dot{\epsilon}=B \sigma^{n} d^{-m} \exp \left(-\frac{E^{*}+p V^{*}}{R T}\right)$,

where $d$ is the grain size, $R$ is the universal gas constant, and $B, n$, $m, E^{*}$, and $V^{*}$ are experimentally determined creep parameters. As diffusion and dislocation creep are the predominant deformation mechanisms in silicates, their creep parameters used for the calculations are listed in Table 3. Owing to the lack of creep activation parameters for lower mantle minerals (e.g., perovskite and post-perovskite), we model the whole mantle viscosity by assuming a dry olivine rheology.

The viscosity $\eta=\sigma /(2 \dot{\epsilon})$ of a given isotropic, incompressible material can be written as

$\eta_{\mathrm{diff}}(d)=\frac{1}{2 B} d^{m} \exp \left(\frac{E^{*}+p V^{*}}{R T}\right)$, 
and

$$
\begin{aligned}
\eta_{\text {disl }}(\sigma) & =\frac{1}{2}\left[\frac{1}{B} \exp \left(\frac{E^{*}+p V^{*}}{R T}\right)\right] \sigma^{1-n} \\
\eta_{\text {disl }}(\dot{\epsilon}) & =\frac{1}{2}\left[\frac{1}{B^{1 / n}} \exp \left(\frac{E^{*}+p V^{*}}{n R T}\right)\right] \dot{\epsilon}^{(1-n) / n} \\
\eta_{\text {disl }}(\Delta) & =\frac{1}{2}\left[\frac{1}{B^{2 /(n+1)}} \exp \left(\frac{2}{n+1} \frac{E^{*}+p V^{*}}{R T}\right)\right] \Delta^{(1-n) /(n+1)}
\end{aligned}
$$

where the dislocation creep is expressed as a function of either constant stress $\sigma$, strain rate $\dot{\epsilon}$, or viscous dissipation $(\Delta=\sigma \dot{\epsilon})$, respectively. Diffusion creep depends on the grain size, which is typically $4 \mathrm{~mm}$ for the upper and $2 \mathrm{~mm}$ for the lower mantle of the Earth (e.g., Karato 2008). Furthermore, we assume for (grain-size-independent) dislocation creep a constant stress of $1 \mathrm{MPa}$, a constant strain rate of $10^{-15} \mathrm{~s}^{-1}$, and a constant viscous dissipation of $10^{-9} \mathrm{~Pa} \mathrm{~s}^{-1}$ (Ranalli 2001), which are the values typically used to model the viscosity profile of the Earth. We calculate the dominant creep rate at any depth as the minimum possible effective viscosity $\eta_{\mathrm{eff}}=\min (\eta)$ (e.g., Ranalli 2001).

Substituting Eq. (11) into (10) and introducing the local Nusselt number (Wagner et al. 2011a)

$N u_{r}=\left(1+\frac{k_{v}}{k_{c}}\right)\left[1-\frac{k_{v}}{q}\left(\frac{\mathrm{d} T}{\mathrm{~d} r}\right)_{S}\right]^{-1}$

leads us to an equation for the temperature $T(r)$

$$
\frac{\mathrm{d} T}{\mathrm{~d} r}=-\frac{q}{N u_{r} k_{c}},
$$

where $r$ is the radial distance from the center of the planet. The dimensionsless Nusselt number $\mathrm{Nu}$ describes the local ratio of total heat flux to conductive heat flux. In the absence of convection $\left(k_{v}=0\right.$, see Eq. (12)), $N u$ equals 1 , while it increases with increasing vigor of convection. Additionally, the total steady state heat flux $q(r)$ within the silicate mantle shell is calculated from

$\frac{\mathrm{d} q}{\mathrm{~d} r}=\epsilon_{m} \rho-2 \frac{q}{r}$

where $\epsilon_{m}$ is the specific heat production rate.

Since the viscosity of metallic alloys under planetary core conditions are not well-constrained, we assume a fully adiabatic radial temperature distribution $T(r)$ within the iron core and replace Eq. (19) with

$\frac{\mathrm{d} T}{\mathrm{~d} r}=-\frac{\gamma \rho g}{K_{S}} T$

We solve the implicit system of thermal equations (Eqs. (19)-(21)) simultaneously with the mechanical equations (Eqs. (3)-(6)) by numerical integration using a BDF algorithm. Boundary conditions are given as follows: (a) central boundary conditions at $r=0$ are $q(0)=0$ and $T(0)=T_{\mathrm{c}}$, where $T_{\mathrm{c}}$ is a chosen starting guess for the central temperature; and (b) surface boundary conditions at $r=R_{\mathrm{p}}$ are $q\left(R_{\mathrm{p}}\right)=q_{\mathrm{s}}$ and $T\left(R_{\mathrm{p}}\right)=T_{\mathrm{s}}$. The integration again stops if all boundary conditions are met.

\subsection{Thermodynamic properties}

Two important thermodynamic quantities, which are often assumed to be constant in terrestrial mantles, are the thermal expansivity and the thermal conductivity. The thermal expansivity $\alpha$ depends on temperature and pressure according to

$\alpha(T, p)=\alpha(T) x^{\delta_{T}}$,
Table 4. Thermal conductivity at ambient conditions, thermal expansion coefficients, and Anderson-Grüneisen parameter for two mantle species.

\begin{tabular}{lcccc}
\hline \hline Mineral & $\begin{array}{c}k_{0} \\
{\left[\mathrm{~W} \mathrm{~K}^{-1} \mathrm{~m}^{-1}\right]}\end{array}$ & $\begin{array}{c}\alpha_{1} \\
{\left[10^{-5} \mathrm{~K}^{-1}\right]}\end{array}$ & $\begin{array}{c}\alpha_{2} \\
{\left[10^{-8} \mathrm{~K}^{-2}\right]}\end{array}$ & $\delta_{T}$ \\
\hline $\mathrm{MgSiO}_{3}{ }^{a}$ & $4.7^{c}$ & 2.6 & 1.0 & 6.5 \\
$\mathrm{Mg}_{2} \mathrm{SiO}_{4}{ }^{b}$ & $5.2^{d}$ & 2.69 & 2.12 & 8.4 \\
\hline
\end{tabular}

Notes. The temperature dependence of the thermal expansivity is expressed in linear form as $\alpha(T)=\alpha_{1}+\alpha_{2} T$.

References. ${ }^{(a)}$ Katsura et al. (2009b); ${ }^{(b)}$ Katsura et al. (2009a); ${ }^{(c)}$ Hofmeister (1999); ${ }^{(d)}$ Horai (1971).

where $\delta_{T}$ is the Anderson-Grüneisen parameter accounting explicitly for the pressure dependence. Table 4 lists the parameters applied to the upper olivine mantle and the subjacent perovskite layer.

Owing to the lack of experimental data for the postperovskite mineralogical phase, we evaluate the thermal expansivity locally using the definition of the thermodynamic Grüneisen parameter

$\gamma=\frac{\alpha K_{S}}{\rho C_{\mathrm{p}}}$,

where $K_{S}$ is the adiabatic bulk modulus and $C_{\mathrm{p}}$ is the specific heat capacity. Both quantities are directly obtained from the equation of state of post-perovskite.

The thermal conductivity $k_{\mathrm{c}}$ is a bulk material property that can be written as the sum of the phonon contribution $k_{\text {lat }}$, the radiative contribution $k_{\mathrm{rad}}$, and the electronic contribution $k_{\mathrm{el}}$

$k_{\mathrm{c}}(T, p)=k_{\mathrm{lat}}(T, p)+k_{\mathrm{rad}}(T)+k_{\mathrm{el}}(T)$.

Owing to the elevated pressure conditions in the deep interior of terrestrial exoplanets, the phonon contribution is expected to be the dominant part of the thermal conductivity. According to Hofmeister (1999), the thermal conductivity due to lattice vibrations is given by

$k_{\text {lat }}=k_{0}\left(\frac{298}{T}\right)^{b} \exp \left[-\left(4 \gamma+\frac{1}{3}\right) \int_{298}^{T} \alpha(\theta) \mathrm{d} \theta\right]\left(1+K_{0}^{\prime} \frac{p}{K_{0}}\right)$,

where the exponent $b$ depends on the type of chemical bonding ( $\sim 0.33$ for most mantle minerals) and $k_{0}$ is the thermal conductivity at ambient conditions. The values used for this parameterization are summarized in Table 4. Furthermore, we assume that post-perovskite has a similar thermal conductivity as perovskite at ambient conditions. This assumption arises from the lack of measurement data for post-perovskite in the literature.

The smaller, pressure-independent radiative conductivity, accounting for only $10 \%$ of the total thermal conductivity at temperatures prevailing at the Earth's D" layer (Goncharov et al. 2008), has been neglected. We also neglect the conductivity component from electron transport, which would contribute only in the case of strongly elevated temperatures (van den Berg et al. 2010).

\subsection{Two-dimensional mantle convection simulations}

The depth variations of the thermodynamic and transport parameters evaluated with our structural models can have a strong influence on the interior dynamics of the exoplanets under study. 
Table 5. Definition of the non-dimensional numbers used in the convection simulations for CoRoT-7b, model case C3.

\begin{tabular}{lc}
\hline \hline $\begin{array}{l}\text { Non-dimensional numbers } \\
\text { based on averaged parameters }\end{array}$ & $\begin{array}{c}\text { Non-dimensional numbers } \\
\text { based on surface parameters }\end{array}$ \\
\hline $\bar{R} a=\frac{\bar{\rho}^{2} \bar{\alpha} \bar{g} \overline{c_{\mathrm{p}}} \Delta T D_{m}^{3}}{\eta_{\mathrm{s}} \overline{k_{\mathrm{c}}}}$ & $R a_{\mathrm{s}}=\frac{\rho_{\mathrm{s}}^{2} \alpha_{\mathrm{s}} \bar{g} \overline{c_{\mathrm{p}}} \Delta T D_{m}^{3}}{\eta_{\mathrm{s}} k_{\mathrm{cs}}}$ \\
$\bar{R} a_{\epsilon}=\frac{\bar{\rho}^{3} \bar{\alpha} \bar{g} \overline{c_{\mathrm{p}}} \epsilon_{m} D_{m}^{5}}{\eta_{\mathrm{s}} \bar{k}_{\mathrm{c}}^{2}}$ & $R a_{\epsilon \mathrm{s}}=\frac{\rho_{\mathrm{s}}^{3} \alpha_{\mathrm{s}} \bar{g} \overline{c_{\mathrm{p}}} \epsilon_{m} D_{m}^{5}}{\eta_{\mathrm{s}} k_{\mathrm{cs}}^{2}}$ \\
$\bar{D} i=\frac{\bar{\alpha} \bar{g} D_{m}}{\overline{c_{\mathrm{p}}}}$ & $D i_{\mathrm{s}}=\frac{\alpha_{\mathrm{s}} \bar{g} D_{m}}{\overline{c_{\mathrm{p}}}}$ \\
\hline
\end{tabular}

Notes. The bar is used to indicate the radial average of a given parameter, while the subscript $s$ refers to the corresponding surface values.

Thus, we carried out selected numerical simulations of thermal convection designed to investigate the characteristic mantle flow planform of CoRoT-7b and Kepler-10b. We employed the finitevolume code Gaia (e.g., Hüttig \& Breuer 2011) to solve the conservation equations of mass, momentum, and thermal energy for a creeping fluid with depth-dependent viscosity in a twodimensional (2-D) cylindrical shell.

We introduce three non-dimensional quantities that characterize our convective systems (Table 5): the thermal Rayleigh number $R a$, the Rayleigh number for internal heat sources $R a_{\epsilon}$, and the dissipation number $D i$. The parameter $R a$ measures the vigor of convection in a fluid heated from below and subject to a temperature drop of $\Delta T=T_{\mathrm{cmb}}-T_{\mathrm{s}}, R a_{\epsilon}$ measures the contribution of radiogenic heat production to the convective vigor, and $D i$ is indicative of the degree of compressibility.

Two series of simulations were performed. In both of them, we assumed a depth-dependent viscosity according to the profiles obtained from Eqs. (14)-(17) as shown in Fig. 5. In the first series of runs, we solved the conservation equations under the extended Boussinesq approximation (EBA) (King et al. 2010). The EBA distinguishes itself from the well-known Boussinesq approximation simply in that adiabatic heating and viscous dissipation are taken into account in the energy conservation equation. The density is assumed to be constant except in the buoyancy term so that the fluid is still incompressible. In our EBA simulations, the thermal expansivity $\alpha$, thermal conductivity $k_{\mathrm{c}}$, and reference density $\rho$ have been held constant and $R a, R a_{\epsilon}$, and $D i$ have been calculated using radially averaged values of all pertinent parameters except the viscosity, which was allowed to vary with depth and whose surface value was used (see Fig. 8 panel (a)).

In the second series of model runs, we adopted a compressible formulation, the so-called truncated anelastic liquid approximation (TALA) (King et al. 2010). When using TALA, we accounted for, in addition to viscosity, the depth-dependence of $\alpha, k_{\mathrm{c}}$, and $\rho$ (Fig. 8 panel (b)), hence used their surface values to calculate the two Rayleigh numbers and dissipation number. Since the depth-variations of $C_{\mathrm{p}}$ and $g$ are relatively small, these parameters were assumed to be constant and set to their average values. In Table 5, we report the definitions of $R a, R a_{\epsilon}$, and $D i$ employed for CoRoT-7b (case C3) for both the EBA and TALA runs.

\section{Results}

\subsection{Scaling laws for massive Earth analogs}

To explore CoRoT-7b and Kepler-10b in a wider context and understand how both exoplanets differ from Earth, we initially use the four-layer structural model approach to derive scaling laws for massive Earth-like planets. We fix mineralogy, bulk composition, radiogenic heat sources, and surface properties (pressure and temperature) to values known from the Earth. Figure 1 shows the calculated radial distributions of hydrostatic pressure, temperature, gravitational acceleration, and density for generic Earth-like planets with masses of 1, 2.5, 5, 7.5, $10,12.5$, and $15 M_{\oplus}$, respectively. For comparison, we also show the preliminary reference Earth model (PREM) taken from Dziewonski \& Anderson (1981) and a reference geotherm according to Stacey \& Davis (2008).

Panel (a) of Fig. 1 shows that the relative amount of the highpressure phase of $\mathrm{MgSiO}_{3}$ (post-perovskite) rapidly increases with planetary size. Therefore, the stability field of $\mathrm{MgSiO}_{3}$ post-perovskite is of particular importance for understanding and modeling the interior of massive Earth-like planets. From panel (a), it can be seen that the pressure at the core-mantle boundary of the $7.5 M_{\oplus}$ model exceeds the possible breakdown pressure $(\sim 0.9 \mathrm{TPa})$ for $\mathrm{MgSiO}_{3}$ post-perovskite into $\mathrm{MgSi}_{2} \mathrm{O}_{5}$ and $\mathrm{MgO}$ (Umemoto \& Wentzcovitch 2012). None of the calculated models exhibit a core-mantle boundary pressure higher than $2 \mathrm{TPa}$, where the theoretically predicted entire decomposition into $\mathrm{MgO}$ and $\mathrm{SiO}_{2}$ would occur. Thus, pressure-induced dissociation may play a significant role in Earth-like planets with masses higher than $7.5 M_{\oplus}$.

Panel (b) of Fig. 1 shows that planets more massive than the Earth exhibit generally hotter interiors than previous model calculations (Valencia et al. 2006). The reason for this finding is a super-adiabatic temperature rise, which dominates within the deep mantle of increasingly massive planets. We mainly attribute this increasingly non-adiabatic temperature distribution to a self-regulating mechanism, which partly compensates for the pressure-induced viscosity rise to maintain at least a sluggish convective regime within the deep mantle interior (Wagner et al. 2011a). This viscosity balancing effect is similar to the Tozer effect, which is well-known for the Earth (Tozer 1972). According to this, a higher temperature decreases the mantle strength and leads to more vigorous convection, which coincides with a larger melt production that more efficiently degasses volatiles from the mantle and again increases its strength.

According to panel (c) of Fig. 1, it becomes obvious that the radial distribution of the gravitational acceleration of massive Earth-like planets shows the typical features of terrestrial planets. The surface gravity of exoplanets is an important quantity because it can be derived from a combination of transit photometry and Doppler spectroscopy. Although both measurements have to be made, the advantage of the surface gravity is that it can be derived independently of the mass of the parent star, which is one of the largest sources of error. Consequently, the surface gravity and hence the $\log -g$ contain fewer systematic errors. A power-law fit to the calculated surface gravity as a function of total mass yields a scaling exponent of 0.47 . This scaling relation is used to produce a log- $g$ diagram, which is discussed below.

Panel (d) of Fig. 1 illustrates that the most significant density change occurs at the core-mantle boundary, which represents a chemical material transition from iron to silicate. Owing to the similar elastic properties, the deep mantle phase transition between post-perovskite and perovskite is hardly visible. A more pronounced density discontinuity is located between the two uppermost layers, where the mineralogical transition between $\mathrm{MgSiO}_{3}$ perovskite and $\mathrm{Mg}_{2} \mathrm{SiO}_{4}$ (olivine) occurs.

The Earth is used as an anchor point. Thus, we optimized our model approach to reproduce reference Earth data where 

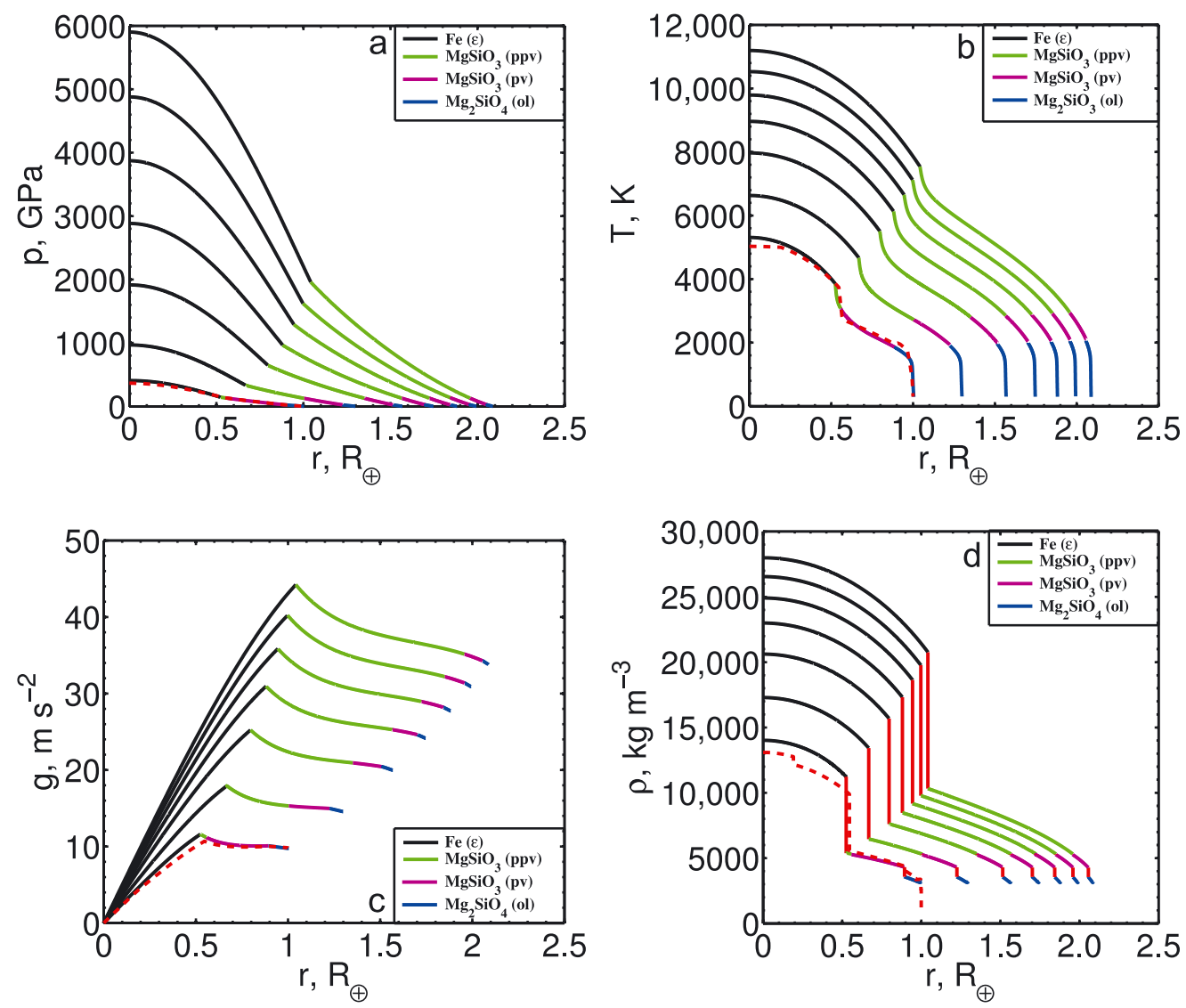

Fig. 1. Radial profiles of a) pressure, b) temperature, c) gravity, and d) density for generic Earth-like exoplanets ranging from 1 (lowermost curve) to $15 M_{\oplus}$ (uppermost curve): the four compositional layers are represented by different colors, while the solid red lines in panel d) indicate discontinuities caused by pressure-induced phase transitions or step-wise compositional changes. The dashed red lines denote a reference Earth model.

the $1 M_{\oplus}$ planet is as close as possible (see Fig. 1). A comparison between the $1 M_{\oplus}$ and the reference Earth model indicates that our modeling approach is capable of producing satisfactory results. Marginal discrepancies between the calculated distributions and the reference profiles are likely caused by simplifying assumptions such as the applied mineralogical and chemical model, uncertainties associated with the local viscosity (e.g., grain size distribution), and the lack of a liquid outer core which is known to exist on Earth.

The interior structures and corresponding scaling laws of rocky planets more massive than the Earth are governed by the equation of state and compositional details. To quantify the uncertainties caused by extrapolation, Wagner et al. (2011a) compared several equations of state for the radial distribution of density to each other. As a result, they found that the predicted planetary radii of rocky exoplanets of up to $10 M_{\oplus}$ differ by less than $2 \%$ among the EoS considered, which is well within current observational limits. The effects of light elements within the core or a different mantle composition were discussed by Valencia et al. (2006). Structural models with a fully molten core can be found in Sotin et al. (2007).

The thermal state evaluated according to the mixing-length approach is significantly influenced by the choice of creep activation parameters (Wagner et al. 2011b). A remaining challenge arises from the limited experimental data of $\mathrm{MgSiO}_{3}$ postperovskite, which determines the radial temperature distribution within the deep interior produced by viscosity and influences the lower mantle heat transport due to thermal conductivity.
The corresponding scaling relationships for massive Earth analogs are shown in Fig. 2. The internal hydrostatic pressure taken at the center or the core-mantle boundary scales linearly with the total mass as seen in panel (a) of Fig. 2. The calculated results obtained for the two CoRoT-7b cases $(\mathrm{C} 1, \mathrm{C} 3)$ and Kepler-10b (K1) are indicated by triangles and stars, respectively. It can be seen that $\mathrm{C} 1$ lies close to the fitting curve for planets with an Earth-like bulk composition characterized by an iron-core mass fraction of $32.6 \mathrm{wt}-\%$. The more massive CoRoT-7b case $\mathrm{C} 3$ in addition to $\mathrm{K} 1$ have higher core pressures owing to their larger iron-core sizes, but to satisfy the measured radii, thinner silicate mantles are required. Hence, lower pressures are reached at the core-mantle boundary.

Panel (b) of Fig. 2 shows the scaling laws obtained for the internal temperature distribution. We observe that the temperature at the center and the core-mantle boundary follows a more complicated power-law relationship, which is governed by the superadiabatic rise within the deep mantle region and creep activation parameters such as grain size and activation volume. Comparing the temperature scaling relations with the relative positions of the CoRoT-7b and Kepler-10b models, we find a picture similar to that already discussed before, in which $\mathrm{C} 1$ represents a scaled-up version of the Earth and both $\mathrm{C} 3$ and $\mathrm{K} 1$ have higher central temperatures and lower temperatures at the core-mantle boundary.

Our scaling laws for the mantle thickness and the mean mantle density are shown in panels (c) and (d) of Fig. 2, respectively. Both quantities are fitted with a power-law relationship. For the 

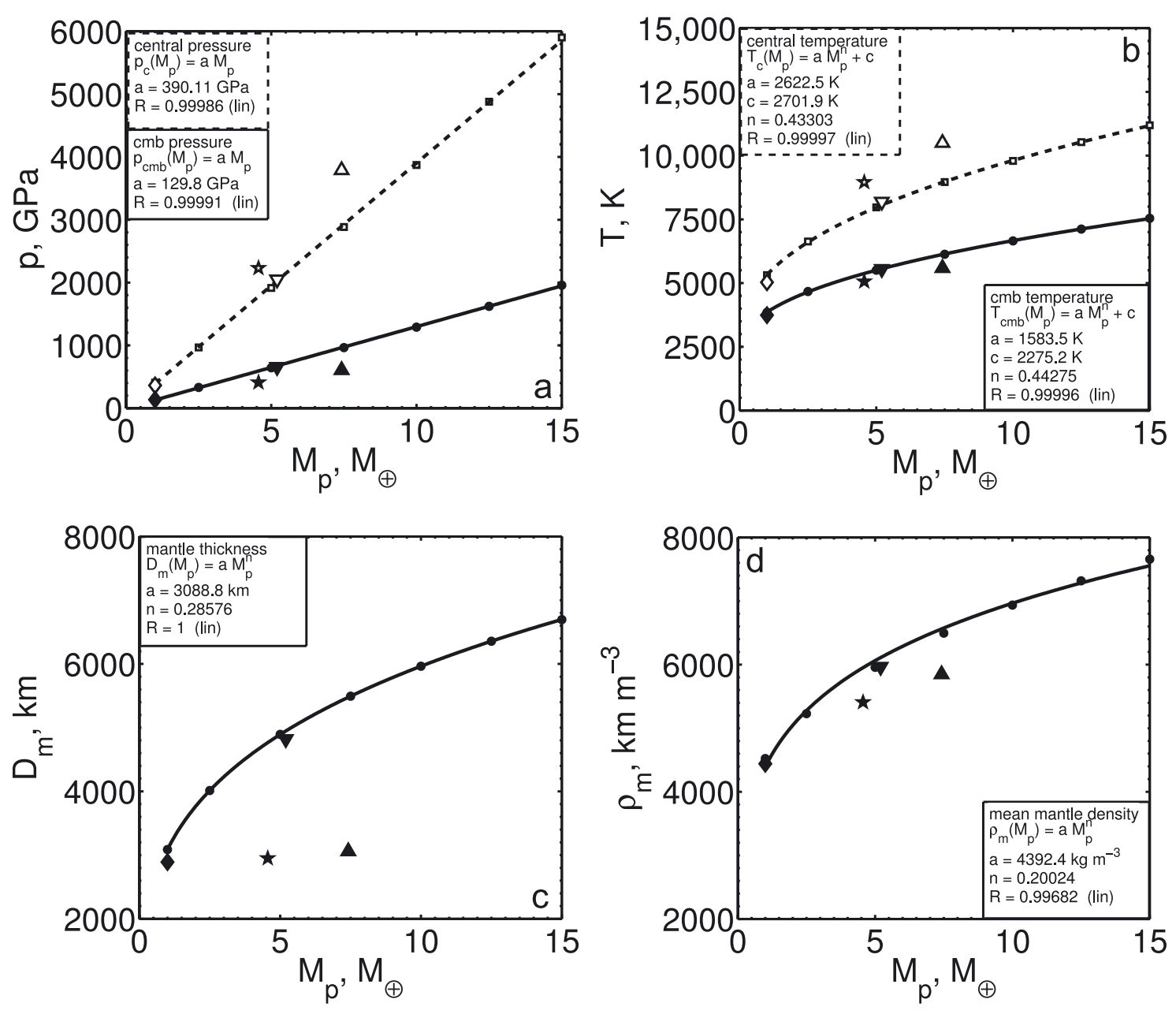

Fig. 2. Scaling laws for characteristic quantities of Earth-like exoplanets in the range of $1-15 M_{\oplus}$ : a) central and core-mantle boundary pressure, b) central and core-mantle boundary temperature, $\mathbf{c}$ ) mantle thickness, and d) mean mantle density. The black diamond denotes the position of the Earth. The two CoRoT-7b cases $\mathrm{C} 1$ and $\mathrm{C} 3$ are labeled by the triangles and the Kepler-10b case K1 is marked as a star. We do not show the case of $\mathrm{C} 2$, because its values do not significantly differ from $\mathrm{C} 1$.

mantle thickness, we find a scaling exponent of 0.29 , which compares well with the range of $0.285-0.295$ previously proposed by Valencia et al. (2006). For the mean mantle density, the obtained scaling exponent of 0.20 closely agrees with the values of 0.19-0.20 found by Valencia et al. (2006). The robustness of these scaling relationships is due the large-scale structure of the interior being governed by both pressure and material properties, but not the radial temperature distribution (Valencia et al. 2009). The $\mathrm{C} 1$ model lies close to the calculated scaling relationships for solid exoplanets with an Earth-like bulk composition. For C3 and $\mathrm{K} 1$, calculations yield smaller mantle thicknesses than the obtained scaling relationship, owing to larger iron cores, which are a consequence of the higher average densities.

Rocky exoplanets without a hydrogen-helium atmosphere are expected to have a planetary radius in the range of $1.25-2 R_{\oplus}$ (Borucki et al. 2011). Figure 3 relates the surface gravity to the total radius of fully differentiated planetary bodies with an Earth-like bulk composition (solid curve) and homogeneous, self-compressible spheres of different materials (dashed curves). The latter represent unlikely extremal cases, but illustrate the possible range of compositions for solid exoplanets. This socalled $\log -g$ diagram is of particular interest to planetary astronomers, since it offers the opportunity to infer a planet's bulk composition from its surface gravity, which can be accurately

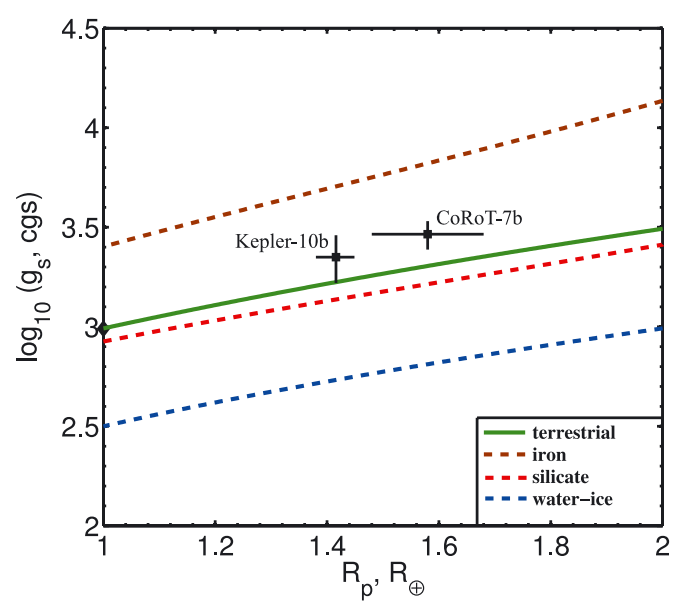

Fig. 3. The logarithm of the surface acceleration as a function of planetary radius (log- $g$ diagram) for Earth-like exoplanets (green curve) corresponding to planetary sizes of $1-2 R_{\oplus}$. To define boundaries and illustrate the calculated trends, the expected $\log _{-} g$ for homogeneous, self-compressible spheres of iron, silicate, and high-pressure water ice have been added as dashed lines. The black diamond represents the position of the Earth. The crosses denote the relative position of CoRoT-7b (C3) and Kepler-10b (K1), respectively, and indicate their observational uncertainties. 

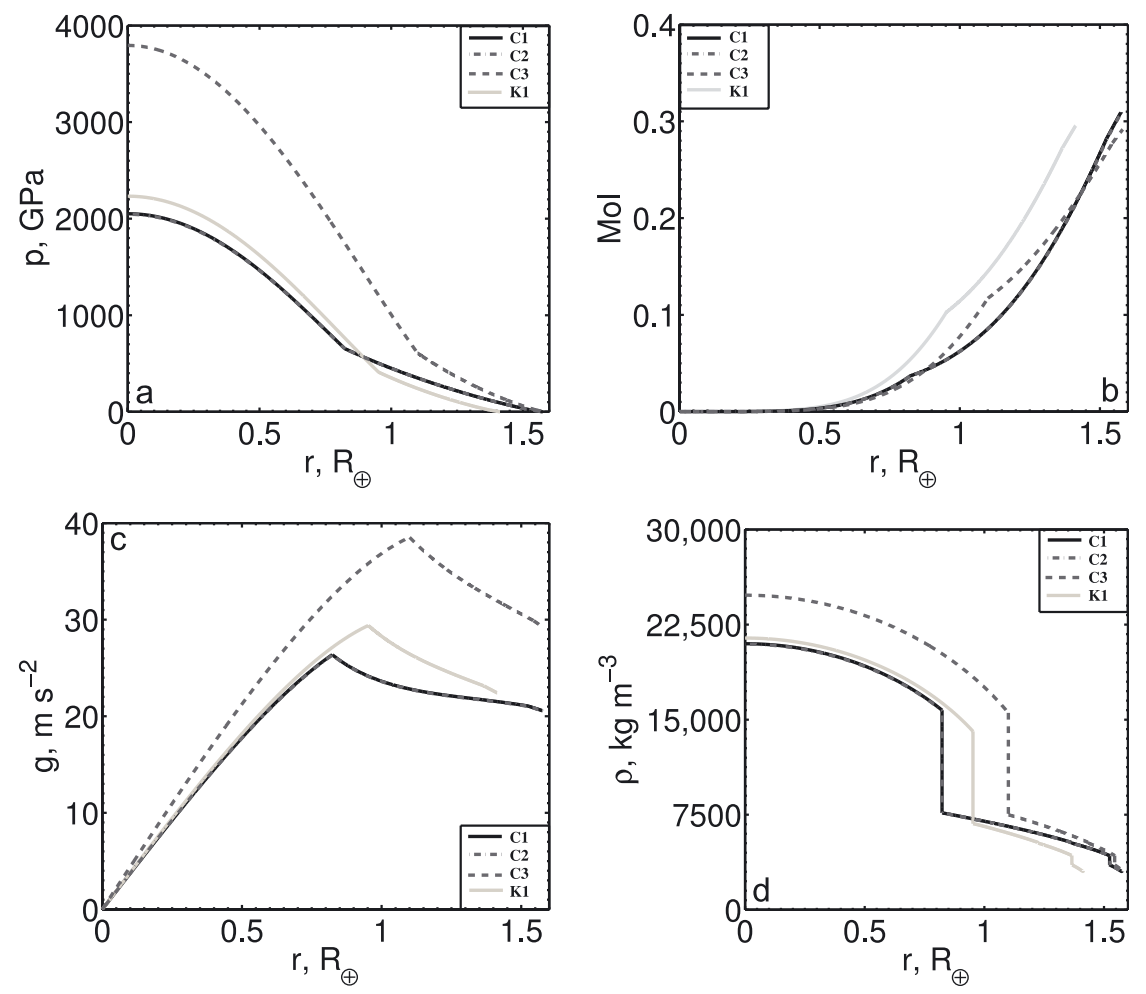

Fig. 4. Depth-dependent interior structure of CoRoT-7b and Kepler-10b. From upper left to lower right, the panels illustrate the radial distribution of a) hydrostatic pressure, b) dimensionsless moment-of-inertia (MoI) factor, c) acceleration of gravity, and d) density, respectively. The line style indicates the different cases discussed in the text. The model case $\mathrm{C} 2$ plots on top of $\mathrm{C} 1$. obtained from precise transit photometry (e.g., PLATO) combined with Doppler spectroscopy according to the relation

$g_{\mathrm{s}}=\frac{2 \pi}{P} \frac{\sqrt{1-e^{2}} K_{\star}}{\left(R_{\mathrm{p}} / a\right)^{2} \sin i}$

where $e$ is the eccentricity, $i$ is the inclination, and $K_{\star}$ is the radial-velocity semi-amplitude (Winn 2011).

When comparing the positions of Kepler-10b and CoRoT-7b to those of generic terrestrial planets (green curve), it becomes clear that both exoplanets are sufficiently compact to be classified as rocky bodies. Both exoplanets appear to be situated on a straight line located above that for massive Earth analogs, pointing towards similar bulk compositions with a comparable high iron content or relatively large iron cores, depending on their internal differentiation. A similar result was obtained by Valencia (2011), suggesting that Kepler-10b is a rocky planet with a bulk composition ranging from Earth-like to Mercury-type, which is more enriched in iron. When considering CoRoT-7b and the planet's controversial nature owing to its different mass estimates (e.g., Pont et al. 2010), we conclude that a rocky composition is the most plausible.

\subsection{Internal structure: CoRoT-7b versus Kepler-10b}

In the following, we use our four-layer structural models to describe in detail the internal structure and composition of CoRoT-7b and Kepler-10b. Figure 4 shows the resulting depthdependent interior structure of the CoRoT-7b cases (C1, C2, C3) and Kepler-10b (K1).

The radial distribution of hydrostatic pressure is shown in panel (a) of Fig. 4. In principle, two characteristic regions are observed for all planetary models: a linear pressure increase from the planet's surface to the core-mantle boundary is followed by a parabolic increase in pressure within the planet's core. Since all cases of CoRoT-7b and Kepler-10b exhibit mantle pressures well below $0.9 \mathrm{TPa}$ at the core-mantle boundary, postperovskite is most likely the predominant mantle mineralogical phase within both exoplanets. Kepler-10b has the lowest pressure at the core-mantle boundary but a higher central pressure than the CoRoT-7b cases $\mathrm{C} 1$ and $\mathrm{C} 2$. This finding can be understood in terms of Kepler-10b's smaller size and its only slightly lower total mass than for the terrestrial CoRoT-7b models $(\mathrm{C} 1$ and C2), which leads to a small planetary mantle but a larger and heavier iron core. The large difference between the radial pressure distributions of the terrestrial CoRoT-7b models $(\mathrm{C} 1$ and $\mathrm{C} 2$ ) and the iron-enriched CoRoT-7b case (C3) results from the significantly higher mass of the latter.

In general, an important quantity for compositional and structural models is the moment of inertia (MoI), which describes how strongly mass is concentrated towards a planet's center. Panel (b) of Fig. 4 shows the radial distribution of the dimensionsless moment-of-inertia factor, which is given by $\theta /\left(M_{\mathrm{p}} R_{\mathrm{p}}^{2}\right)$. Owing to the model assumption of full differentiation, calculations yield relatively small MoI factors of about 0.30 for all model cases. The iron-enriched cases C3 and K1 tend to generate slightly smaller planetary MoI factors because the large iron cores cause a strong concentration of mass towards the core.

The corresponding gravitational acceleration as a function of the radial distance from the planet's center is shown in panel (c) of Fig. 4. Two characteristic regions can again be distinguished in all planetary models. From the calculated surface values of the gravitational acceleration, we observe an almost linear increase throughout the planetary mantle to the core-mantle boundary. From there, the gravitational acceleration decreases linearly within the core. The surface value of the gravitational acceleration reflects a planet's bulk composition. We find therefore comparably large surface values of the gravitational acceleration for the iron-enriched CoRoT-7b case (C3) and Kepler-10b owing to their relatively large and heavy iron cores. Comparably lower surface gravitational acceleration values are observed for the models $\mathrm{C} 1$ and $\mathrm{C} 2$, which have terrestrial bulk compositions. 
F. W. Wagner et al.: Rocky super-Earth interiors

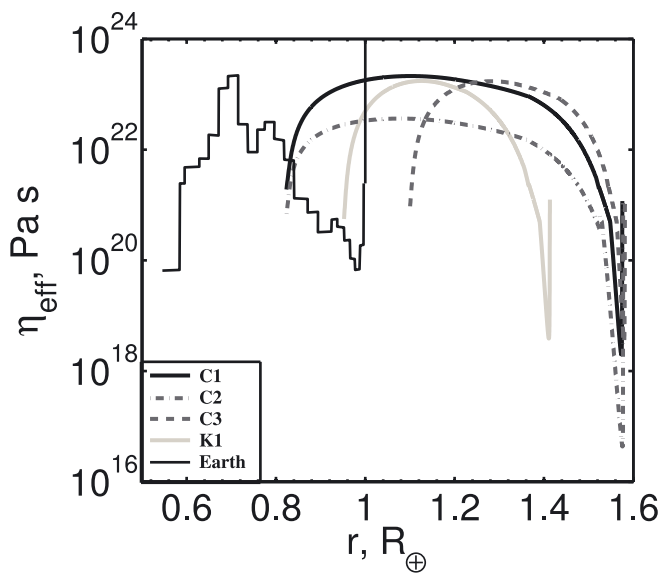

Fig. 5. Effective mantle viscosity for CoRoT-7b and Kepler-10b. The line style indicates the different models discussed in the text. In addition, Earth's mantle viscosity inferred from long-wavelength mantle convection and post-glacial rebound data (Mitrovica \& Forte 2004) is shown for comparison.

The radial density distribution according to the implemented equation of state is plotted in panel (d) of Fig. 4. In all models, the density increases almost linearly with depth within the dominant mantle regions, which are composed of $\mathrm{Mg}_{2} \mathrm{SiO}_{4}$ and $\mathrm{MgSiO}_{3}$, respectively. A density discontinuity occurs at the transition boundary between the upper olivine mantle and the underlying perovskite layer of the lower mantle. Owing to the similar elastic properties of perovskite and post-perovskite (Tsuchiya et al. 2004), the phase transition boundary between the perovskite layer of the lower mantle and the post-perovskite layer of the lowermost mantle is hardly visible. The mantle density increases by a factor of between 2.2 and 2.5 from the surface value of about $3100 \mathrm{~kg} \mathrm{~m}^{-3}$ to the core-mantle boundary. A pronounced density variation is found at the core-mantle boundary, which represents a major material transition from silicate to iron. From there, the density continues to increase with depth parabolically until the planet's center.

The radial mantle viscosity profile is given in Fig. 5. Owing the high surface temperature, which is assumed to be $1300 \mathrm{~K}$, the viscosity close to the planet's surface is on the order of $10^{21} \mathrm{~Pa} \mathrm{~s}$ for all CoRoT-7b cases and Kepler-10b. From this relatively low value, mantle viscosity decreases rapidly by three orders of magnitude to about $10^{18} \mathrm{~Pa}$ s for all models with an Earthlike specific heat production rate, because of a steep temperature gradient within the conductive upper boundary layer. A subsequent increase in viscosity is observed throughout the remaining upper mantle, when convection starts to kick in. Within an extended part of the lower mantle, viscosity stays constant at about $10^{23} \mathrm{~Pa}$ s owing to a viscosity-controlling feedback mechanism involving temperature and pressure. Another large viscosity variation is located near the core-mantle boundary, where another conductive boundary layer separates the silicate mantle from the iron core. The CoRoT-7b case $\mathrm{C} 2$ with an increased specific mantle heat production rate tends to have viscosities, which are in general one order of magnitude lower than those of the other three models. In summary, we expect a vigorously convecting region beneath a thin thermal boundary layer as a result of high surface temperatures and a relatively low viscosity within the upper mantle region. For the lower and lowermost mantle regions, we observe a self-regulating mechanism that balances pressure and the effects of temperature on viscosity. Owing to our choice of creep activation parameters and a
Table 6. Modeling results for CoRoT-7b and Kepler-10b.

\begin{tabular}{lccccc}
\hline \hline & $\mathrm{C} 1$ & $\mathrm{C} 2$ & $\mathrm{C} 3$ & $\mathrm{~K} 1$ & Earth \\
\hline$c m f[\mathrm{wt}-\%]$ & 35 & 35 & 64 & 59.5 & $32.6^{a}$ \\
$M o I_{\mathrm{p}}$ & 0.310 & 0.309 & 0.292 & 0.296 & $0.331^{b}$ \\
$R_{\mathrm{c}}[\mathrm{km}]$ & 5250 & 5250 & 7010 & 6070 & $3480^{c}$ \\
$D_{m}[\mathrm{~km}]$ & 4820 & 4820 & 3060 & 2950 & $2890^{c}$ \\
$p_{\mathrm{cmb}}[\mathrm{GPa}]$ & 656 & 654 & 607 & 410 & $136^{c}$ \\
$p_{\mathrm{c}}[\mathrm{GPa}]$ & 2050 & 2050 & 3790 & 2230 & $364^{c}$ \\
$g_{\mathrm{s}}\left[\mathrm{m} \mathrm{s}^{-2}\right]$ & 20.6 & 20.5 & 29.1 & 22.4 & $9.83^{c}$ \\
\hline
\end{tabular}

Notes. Values of the Earth are given for comparison.

References. (a) Turcotte \& Schubert (2002); (b) Romanowicz \& Lambeck (1977); ${ }^{(c)}$ Dziewonski \& Anderson (1981).

grain size distribution based on an Earth-like mantle, we find that the upper mantle is dominated by dislocation creep, while the lower mantle is mainly controlled by diffusion.

Table 6 summarizes the main modeling results of the interior structure for the CoRoT-7b cases and Kepler-10b. By comparing the case $\mathrm{C} 1$ to $\mathrm{C} 2$, it becomes obvious that the radiogenic heat production rate has a negligible effect on a planet's internal structure. It can also be seen from Table 6 that the ironenriched model (C3) for CoRoT-7b yields a similar structure and bulk composition as Kepler-10b (K1) when comparing the mantle thickness and iron-core mass fraction, respectively. Owing to the relatively high mean densities of the iron-enriched model cases C3 and Kepler-10b, both planets have extended iron cores but rather thin mantles. The calculations also yield mantle thicknesses on the order of about $3000 \mathrm{~km}$ for C3 and K1, which are similar to the thickness of the Earth's silicate mantle. This may be interesting for mantle convection studies because the Rayleigh number strongly scales with the mantle thickness.

\subsection{Thermal state of CoRoT-7b and Kepler-10b}

The radial distribution of temperature is illustrated in Fig. 6. From a fixed surface value of $1300 \mathrm{~K}$, the temperature rapidly increases in all model cases across the thermal lithosphere, where heat is transferred by conduction only. In comparison to the models shown in panel (b) of Fig. 1, the thermal boundary layer is significantly thinner because of the high surface temperature. A subsequent temperature rise is observed throughout the convecting mantle for all CoRoT-7b cases and Kepler-10b becomes a small conductive lid in the lowermost mantle region where the chemical transition between the silicate mantle and the iron core takes place. From the core-mantle boundary, the temperature rises adiabatically throughout the convecting core. It is seen that the adiabatic temperature gradient within the lower mantle is steeper for the most massive case $\mathrm{C} 3$, which has a comparably large iron-core mass fraction primarily due to the larger effect of pressure on viscosity. A similar observation holds for Kepler-10b. As a result of the steeper adiabatic temperature gradient for the iron-enriched models $\mathrm{C} 3$ and $\mathrm{K} 1$, both planets are in general hotter than the terrestrial cases $\mathrm{C} 1$ and $\mathrm{C} 2$. Furthermore, when comparing $\mathrm{C} 1$ to $\mathrm{C} 2$, a five times higher concentration of radiogenic elements in the silicate mantle layer leads to slightly higher temperatures by only a few hundred Kelvin. Table 7 lists the main modeling results for the thermal states of the CoRoT-7b cases and the Kepler-10b model.

Two important thermodynamic quantities governing the heat transport in planetary mantles are the thermal conductivity and 


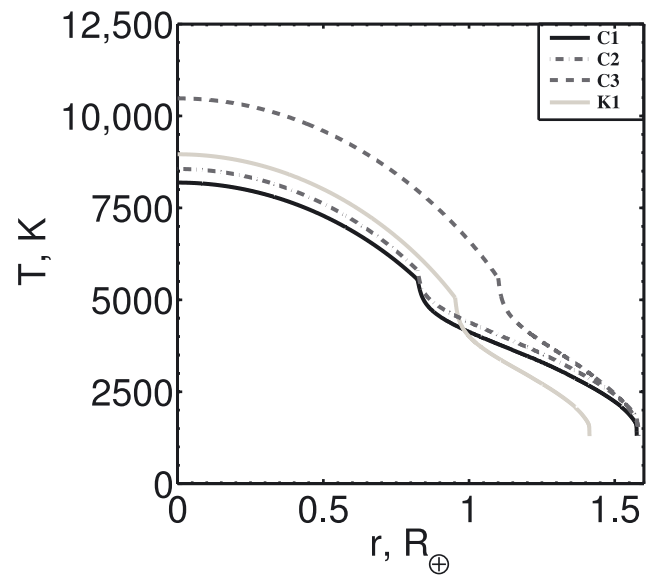

Fig. 6. Depth-dependent temperature structure of CoRoT-7b and Kepler-10b. The line style indicates the different models discussed in the text.

Table 7. Modeling results for CoRoT-7b and Kepler-10b.

\begin{tabular}{lccccc}
\hline \hline & $\mathrm{C} 1$ & $\mathrm{C} 2$ & $\mathrm{C} 3$ & $\mathrm{~K} 1$ & Earth \\
\hline$T_{\mathrm{cmb}}[\mathrm{K}]$ & 5550 & 5800 & 5590 & 5060 & $3740^{a}$ \\
$T_{\mathrm{c}}[\mathrm{K}]$ & 8190 & 8560 & 10,500 & 8960 & $5030^{a}$ \\
$q_{\mathrm{s}}\left[\mathrm{mW} \mathrm{m}^{-2}\right]$ & 129 & 599 & 123 & 106 & $65^{b}$ \\
$q_{\mathrm{cmb}}\left[\mathrm{mW} \mathrm{m}^{-2}\right]$ & 40.5 & 42.4 & 62.7 & 57.2 & $20^{c}$ \\
\hline
\end{tabular}

Notes. Values of the Earth are given for comparison.

References. ${ }^{(a)}$ Stacey \& Davis (2008); ${ }^{(b)}$ Pollack et al. (1993); ${ }^{(c)}$ Sleep (1990).

the thermal expansivity. Panel (a) of Fig. 7 shows the thermal conductivity as a function of the planetary radius within the mantle region. Throughout the mantle, thermal conductivity increases from a surface value of $2.5 \mathrm{~W} \mathrm{~m}^{-1} \mathrm{~K}^{-1}$ by about a factor of 2.0 to 2.6 depending on the model. Peak values from about 7 to $9 \mathrm{~W} \mathrm{~m}^{-1} \mathrm{~K}^{-1}$ are found within the lowermost mantle region, before dropping back to values from about 5 to $7 \mathrm{~W} \mathrm{~m}^{-1} \mathrm{~K}^{-1}$ at the core-mantle boundary. The two implemented mantle phase transitions are clearly visible, each of them reduce the absolute value of the thermal conductivity because of the different material properties. Overall, the relatively low thermal conductivity in all model cases is caused by the hot interior and only the phonon contribution being taken into account.

Panel (b) of Fig. 7 shows the thermal expansivity as a function of the planetary radius within the mantle region. In all three silicate layers, thermal expansivity decreases sharply with depth, because of the increasing pressure. From surface values of about $7 \times 10^{-5} \mathrm{~K}^{-1}$, the thermal expansivity decreases by more than one order of magnitude in all models. Both mantle phase transitions are again clearly visible, each of them increasing the absolute value of the thermal expansivity by a small amount.

\subsection{Mantle dynamics of hot terrestrial exoplanets}

In Fig. 8, we show two long-term snapshots of the temperature field calculated from convection simulations that employ the structural data of model C3. Thermodynamic properties $\left(\rho, \alpha\right.$, and $\left.k_{\mathrm{c}}\right)$ are constant in panel (a) and depth-dependent in panel (b). Simulation runs that use the data of the other structural models do not exhibit significant qualitative differences and are therefore not shown.
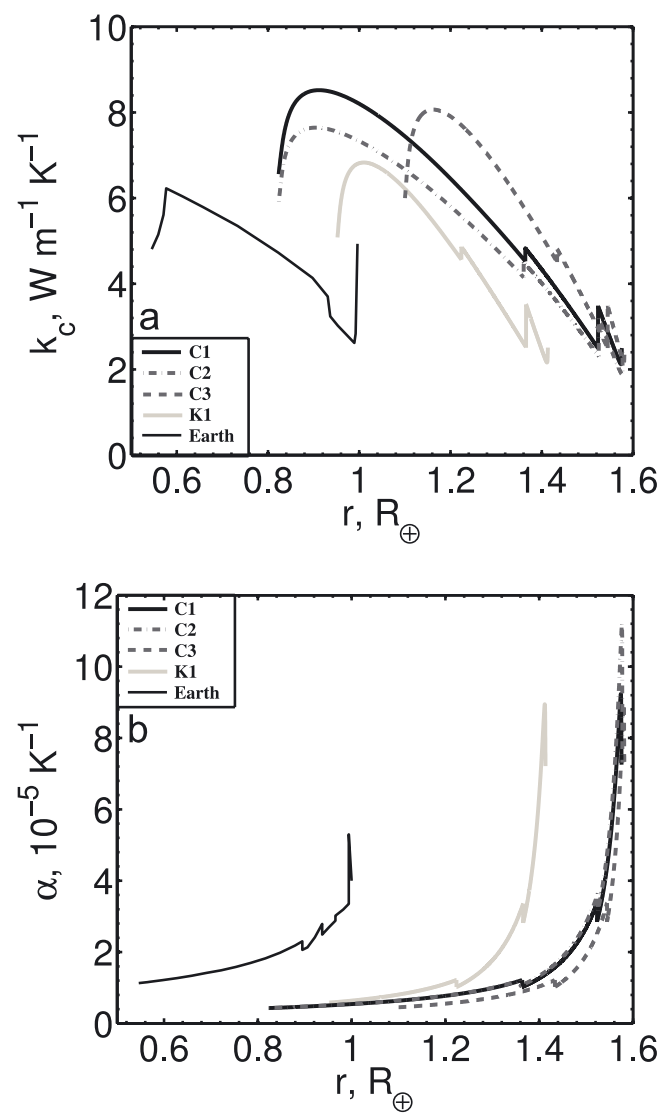

Fig. 7. Thermodynamic mantle properties of CoRoT-7b and Kepler10b: a) thermal conductivity and b) thermal expansivity. The line style indicates the different models discussed in the text. For comparison, we also show the corresponding thermodynamic properties of the Earth according to a) Hofmeister (1999) and b) Stacey \& Davis (2008).

By comparing the two panels, it is evident that the convection pattern is strongly affected by the presence of depthdependent parameters. On the one hand, with constant parameters (panel (a)), convection is sluggish. Hot upwellings forming at the core-mantle boundary have a short lifetime. They do not carry enough buoyancy to rise through the mantle and tend to be sheared and clustered by large-scale flow. Since the largest portion of the mantle is characterized by a high viscosity, which is roughly constant apart from near the core-mantle boundary and surface, downwellings fail to penetrate into the deep mantle and to exhibit the slab-like shape that would be expected for a boundary layer instability. On the other hand, the use of depth-dependent parameters (panel (b)) ensures that upwellings are the most prominent mantle feature. As soon as a thermal instability forms at the core-mantle boundary and starts to rise, it acquires buoyancy during its ascent owing to the growing thermal expansivity with height. The opposite argument applies to downwellings that lose their buoyancy while sinking into the mantle because of the reduction in $\alpha$ with the depth. As a consequence, and also because of the high mantle viscosity, they tend to stagnate in the mid-mantle.

\section{Discussion and conclusions}

The recent discoveries of the first two extrasolar planets with sizes smaller than twice that of Earth have prompted strong interest in their nature and evolution. With the present study, we have calculated the internal structure and thermal state of 

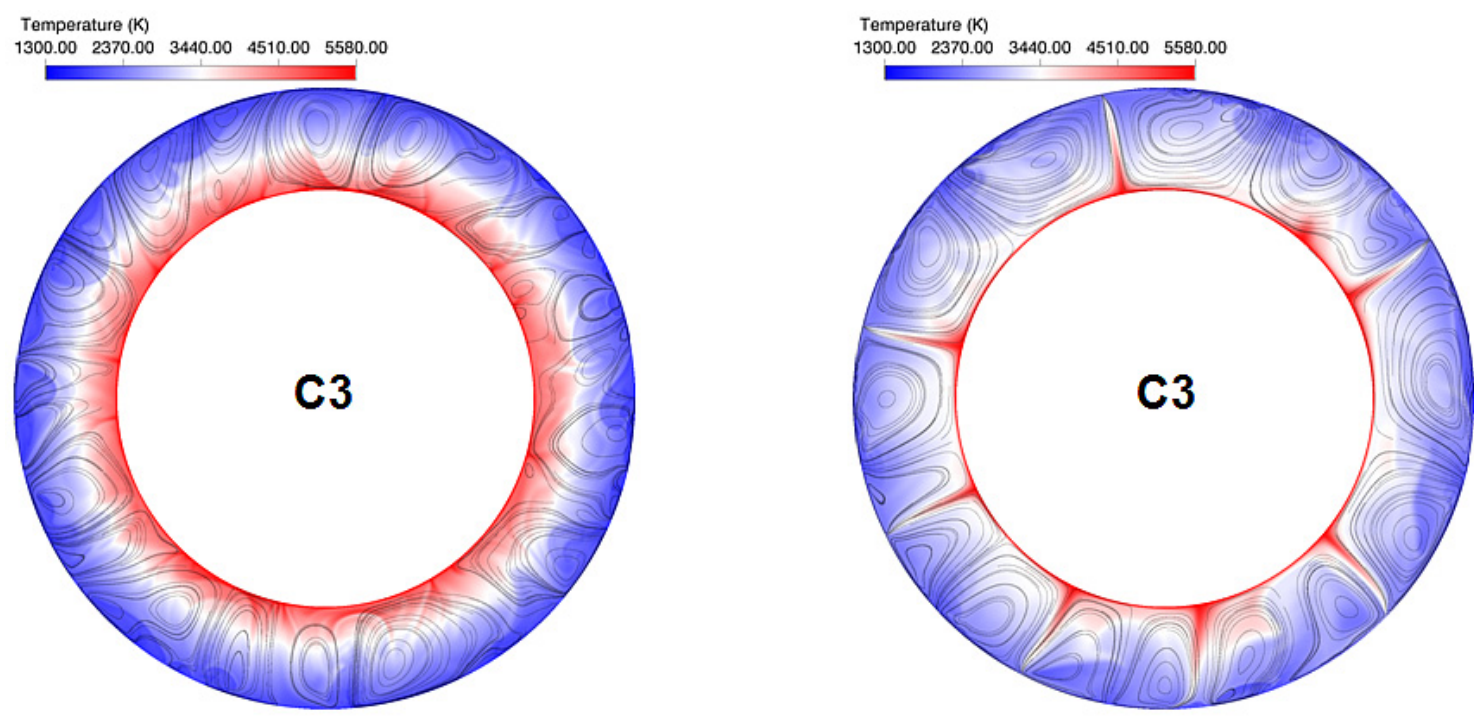

Fig. 8. Mantle convection pattern according to our two-dimensional mantle convection simulations using a) constant and b) depth-dependent thermodynamic properties within the planetary mantle.

CoRoT-7b and Kepler-10b by assuming plausible compositions. Moreover, we have modeled their interior dynamics to determine the key parameters governing the mantle convection pattern in close-in exoplanets with a terrestrial bulk composition. Both planets share many properties, e.g., relatively small planetary radii, comparable total masses, and similar short orbital periods. It is also widely believed that both planets are synchronously rotating around their G-type host stars resulting in extremely high (more than one thousand Kelvin) surface temperatures. Different radiogenic heat production rates within their silicate mantles are suggested by their different ages.

To infer the possible bulk composition of CoRoT-7b and Kepler-10b, a log- $g$ diagram has been used, from which we have been able to classify both planets as rocky. We have then inferred the detailed interior structures of CoRoT-7b and Kepler-10b to compare them with a massive Earth analog. For the measured planetary radius of $1.58 R_{\oplus}$ and a total mass estimate of $5.2 M_{\oplus}$ (Bruntt et al. 2010), CoRoT-7b is expected to have an iron core of 35 wt- $\%$, which is comparable to the Earth's (32.6 wt-\%). Under these circumstances, CoRoT-7b would represent a massive Earth analog. Subsequently, the mass of CoRoT-7b has been subject to discussion and intensely revised, owing to the young and active star CoRoT-7 that causes noise and jitter effects in the radial velocity data. However, a consolidated total mass of $7.42 M_{\oplus}$ has been proposed (Hatzes et al. 2011), for which our calculations imply an iron core of $64 \mathrm{wt}-\%$, which is almost identical in composition to Kepler-10b's 59.5 wt-\%, but more enriched in iron than that of the Earth. Strongly enriched in iron, both planets would be more similar in composition to the planet Mercury than the Earth (e.g., van Hoolst et al. 2007). Studies of thermally-induced atmospheric mass loss strongly suggest that CoRoT-7b and Kepler-10b cannot be the eroded cores of former gas or icy giant planets, but rather formed as rocky planets that have lost only a thin hydrogen atmosphere throughout their entire history (Leitzinger et al. 2011). Nevertheless, we have been unable to address some questions, which therefore remain open, e.g., where did CoRoT-7b and Kepler-10b form? Is it in general possible or perhaps even typical to form iron-rich planets at such close distances to their parent stars? Or is it more likely that CoRoT-7b and Kepler-10b have migrated inwards due to gravitational scattering?
Post-perovskite seems to be the predominant mineralogical phase within the mantles of massive terrestrial exoplanets. Umemoto et al. (2006a) argued based on first principles simulations that $\mathrm{MgSiO}_{3}$ post-perovskite dissociates directly into $\mathrm{MgO}$ and $\mathrm{SiO}_{2}$ at a pressure of around $1 \mathrm{TPa}$. They also predicted that a comparable dissociation process takes place for the $\mathrm{MgSiO}_{3}$ low-pressure analog $\mathrm{NaMgF}_{3}$ at $\sim 40 \mathrm{GPa}$ (Umemoto et al. 2006b), although this has not been observed experimentally (e.g., Grocholski et al. 2010; Martin et al. 2006). This experimental evidence suggests that $\mathrm{MgSiO}_{3}$ post-perovskite either remains stable at pressures largely beyond $1 \mathrm{TPa}$ or undergoes a transition into a yet unidentified post-post-perovskite mineralogical phase. Okada et al. (2010) reported the dissociation of $\mathrm{MnTiO}_{3}$ perovskite into $\mathrm{MnO}$ and $\mathrm{MnTi}_{2} \mathrm{O}_{5}$, which implies that $\mathrm{MgSiO}_{3}$ post-perovskite may not dissociate directly into $\mathrm{MgO}$ and $\mathrm{SiO}_{2}$. The latest results from first principles simulations also suggest a two-stage dissociation for $\mathrm{MgSiO}_{3}$ post-perovskite, in which $\mathrm{MgSiO}_{3}$ dissociates into $\mathrm{MgO}$ and $\mathrm{MgSi}_{2} \mathrm{O}_{5}$ at $\sim 0.9 \mathrm{TPa}$ and subsequently $\mathrm{MgSi}_{2} \mathrm{O}_{5}$ into $\mathrm{MgO}$ and $\mathrm{SiO}_{2}$ at $\sim 2.1 \mathrm{TPa}$ (Umemoto \& Wentzcovitch 2012). Neither the first nor the second dissociation is reached within the mantles of CoRoT-7b and Kepler-10b, therefore the deep interior of both planets is expected to be dominated by $\mathrm{MgSiO}_{3}$ post-perovskite.

Past studies (e.g., Sotin et al. 2007) have suggested that the temperature structure has little effect on the planetary radius. Even for close-in exoplanets such as CoRoT-7b and Kepler-10b that have extremly high surface temperatures, the thermal pressure contribution would be relatively small (Seager et al. 2007). Nevertheless, temperature plays an important role when it comes to the physical state of matter, e.g., liquid or solid, and the thermal evolution of planetary bodies. In the particular case of tidally locked planets without a protecting atmosphere or magnetic shielding, their synchronous orbits may foster extreme surface temperature contrasts of many hundreds to thousand of Kelvin between the irradiated hot dayside and the permanently cold nightside. Recently, van Summeren et al. (2011) have investigated the influence of surface temperature dichotomies on mantle dynamics. Their results strongly emphazise the non-negligible effect of surface temperatures on the convective regime. They also showed that a surface contrast of about $400 \mathrm{~K}$ can already maintain an asymmetric degree- 1 convection 
pattern with plate-like tectonics on the nightside and a continously evolving mobile lid on the dayside. Extreme configurations could even provide enough insulation to melt rocky surfaces and promote the formation of extended oceans of molten rock (Léger et al. 2011).

To include temperature effects, we have applied a mixing length approach to model the thermal structure and properties of planetary mantles. Those calculations have led us to the conclusion of a generally hotter planetary interior than previously thought, implying a not fully adiabatic deep mantle temperature profile. This is explained by the effect of pressure on viscosity, which has to be compensated for by an increasing temperature gradient. The result is a self-regulation process that causes viscosity to increase with depth to less than $10^{23}$ or $10^{24} \mathrm{~Pa} \mathrm{~S}$ in order to prevent the interior from becoming too viscous for convection to proceed. The viscosity structure of the mantles of terrestrial exoplanets remains unclear. For example, recent considerations consistent with mineral physics suggest that viscosity strongly decreases within the deep mantles of super-Earths (Karato 2011), in favor of vigorous convection, whereas a homologous temperature scaling leads to ultra-high viscosities of up to $10^{30} \mathrm{~Pa}$ s (Stamenković et al. 2011), which would be too high for convection to occur.

In previous studies, either the radiative properties of the star (Léger et al. 2011) or the dissipation of tidal energy induced by the gravitational forces of the associated planetary system (Barnes et al. 2010) have been taken into consideration as viable heat sources. To complement those studies, we have investigated the radiogenic heating caused by the decay of long-lived radioactive elements such as $\mathrm{U}, \mathrm{K}$, and $\mathrm{Th}$ as a consequence of the relatively young age of CoRoT-7b. The model calculations indicate that radiogenic heating has a negligible effect on the large-scale internal structure of rocky exoplanets. However, higher mantle temperatures yield a strong reduction in viscosity, which will ultimately lead to vigorous convection, possibly increasing the total rate of volcanic activity. A viscosity reduction caused by radiogenic heating could also enforce the possibly strong tidal effects within close-in exoplanets such as CoRoT-7b and Kepler-10b, affecting their present thermal states of the deep interior, surface geology, and orbital evolution.

A fundamental feature of massive rocky exoplanets is that temperature and pressure conditions in the deep interior vary over a significantly larger range than for the terrestrial bodies of the Solar System. For this reason, the depth-dependence of thermodynamic parameters is expected to become important. To address this point, we have modeled the interior structure and thermal state of CoRoT-7b and Kepler-10b as a case study for close-in exoplanets with a terrestrial bulk composition to obtain the depth-dependent distribution of important thermodynamic properties (e.g., density, thermal expansivity, and thermal conductivity). Our results indicate that the mantle convection pattern of massive terrestrial planets is strongly influenced by the depthdependence of thermodynamic quantities. A key role is played by the strongly decreasing thermal expansivity, which stabilizes hot upwellings as cold downwellings disperse in the mid-mantle region.

A limitation of this study is that neither the electronic nor the radiative contribution of the thermal conductivity have been taken into account. The thermal conductivity profiles of CoRoT-7b and Kepler-10b are altered by three factors:

(a) Radiation (radiative thermal conductivity) and excited electrons (electronic thermal conductivity), both may become significant contributors to the total thermal conductivity at high temperatures (e.g., Stamenković et al. 2011). To assess the order of magnitude of the radiative thermal conductivity in the deep interior of exoplanets, we applied a classical $T^{3}$-law, which is expected to be valid up to $4500 \mathrm{~K}$. For that temperature, we obtained $7.7 \mathrm{~W} \mathrm{~m}^{-1} \mathrm{~K}^{-1}$ according to $k_{\mathrm{rad}}=$ $\chi T^{3}$, where $\chi=8.5 \mathrm{~W} \mathrm{~m}^{-1} \mathrm{~K}^{-4}$ (Hofmeister 1999). This relatively large value is comparable to the calculated values of the thermal conductivity close to the core-mantle boundary. As a result, the temperature-induced decrease in the thermal conductivity near the core-mantle boundary could be largely compensated for by an increasingly high radiative thermal conductivity. Little is known about the electronic thermal conductivity of $\mathrm{MgSiO}_{3}$ except that an increasing electronic conductivity leads to a decreasing radiative thermal conductivity and rather extreme temperatures are needed to excite a sufficient amount of electrons (Umemoto et al. 2006a).

(b) $\mathrm{MgSiO}_{3}$ post-perovskite, which may have a significantly higher thermal conductivity than perovskite. Hunt et al. (2012) measured the thermal diffusivity of the perovskite and post-perovskite phase of $\mathrm{CaIrO}_{3}\left(\mathrm{a} \mathrm{MgSiO}_{3}\right.$ low-pressure analog) at elevated pressures and temperatures. They found that the thermal conductivity of post-perovskite is slightly less than twice that of perovskite, confirming a prediction of Hofmeister et al. (2007). These new findings increase the absolute thermal conductivity values of the post-perovskite layer, but do not alter its dome-shaped profile.

(c) Dissociation of $\mathrm{MgSiO}_{3}$, which ultimately leads to a significant increase in $\mathrm{MgO}$ within planetary mantles. This effect is irrelevant for CoRoT-7b and Kepler-10b because the pressures at the core-mantle boundary are vastly below the breakdown pressure of 0.9 TPa. Nevertheless, $\mathrm{MgO}$ is known to have a relatively high thermal conductivity (e.g., de Koker 2010) and may dominate the transport and thermal properties of rocky planets more massive than $7.5 M_{\oplus}$.

Hence, the total thermal conductivity may be higher than our results suggest, thereby amplifying the strength of mantle plumes and decreasing the tendency to form a layered convection pattern (Matyska \& Yuen 2006; Tosi et al. 2010). Moreover, a substantial layer composed of $\mathrm{MgSiO}_{3}$ post-perovskite may have higher thermal conductivity than $\mathrm{MgSiO}_{3}$ perovskite, thereby increasing the heat flux conducted across the core-mantle boundary with implications for whole mantle convection and thermal evolution (Stamenković et al. 2012).

The quest to find new small-sized exoplanets is an ongoing task. Two new examples for planetary bodies with radii smaller than $2 R_{\oplus}$ have been recently reported by the Kepler team. These are Kepler-11b, a planet with an average compressed density significantly lower than that of the Earth suggesting that its composition is similar to that of the icy giants Uranus and Neptune of the outer Solar System (Lissauer et al. 2011), and Kepler-9d (Holman et al. 2010), a planet for which the mass is still unknown. Nevertheless, theoretical calculations imply that Kepler-9d has a rocky structure with a total mass of 4-16 $M_{\oplus}$ (e.g., Havel et al. 2011).

Acknowledgements. We thank Diana Valencia for her constructive comments and valuable suggestions, which considerably improved the manuscript. This research has been supported by the Helmholtz Association through the research alliance "Planetary Evolution and Life", the Czech Science Foundation (project P210/11/1366), and the German Research Foundation (grant number TO 704/1-1). 


\section{References}

Abe, Y. 1997, Phys. Earth Planet. Inter., 100, 27

Al'tshuler, L. V., Brusnikin, S. E., \& Kuz'menkov, E. A. 1987, J. Appl. Mech. Tech. Phys., 28, 129

Barnes, R., Raymond, S. N., Greenberg, R., Jackson, B., \& Kaib, N. A. 2010, ApJ, 709, L95

Batalha, N. M., Borucki, W. J., Bryson, S. T., et al. 2011, ApJ, 729, 27

Borucki, W. J., Koch, D. G., Basri, G., et al. 2011, ApJ, 736, 19

Bruntt, H., Deleuil, M., Fridlund, M., et al. 2010, A\&A, 519, A51

de Koker, N. 2010, Earth Planet. Sci. Lett., 292, 392

Dewaele, A., Loubeyre, P., Occelli, F., et al. 2006, Phys. Rev. Lett., 97, 215504

Dziewonski, A. M., \& Anderson, D. L. 1981, Phys. Earth Planet. Inter., 25, 297

Goncharov, A. F., Haugen, B. D., Struzhkin, V. V., Beck, P., \& Jacobsen, S. D. 2008, Nature, 456, 231

Grocholski, B., Shim, S. H., \& Prakapenka, V. B. 2010, Geophys. Res. Lett., 37, L14204

Hansen, C. J., Kawaler, S. D., \& Trimble, V. 2004, Stellar Interiors (Springer)

Hatzes, A. P., Dvorak, R., Wuchterl, G., et al. 2010, A\&A, 520, A93

Hatzes, A. P., Fridlund, M., Nachmani, G., et al. 2011, ApJ, 743, 75

Havel, M., Guillot, T., Valencia, D., \& Crida, A. 2011, A\&A, 531, A3

Hofmeister, A. M. 1999, Science, 283, 1699

Hofmeister, A. M., Branlund, J. M., \& Pertermann, M. 2007, Properties of rock and minerals - thermal conductivity of the Earth, in Treatise in Geophysics, ed. G. Schubert (Elsevier)

Holman, M. J., Fabrycky, D. C., Ragozzine, D., et al. 2010, Science, 330, 51

Horai, K. 1971, J. Geophys. Res., 76, 1278

Hunt, S. A., Davies, D. R., Walker, A. M., et al. 2012, Earth Planet. Sci. Lett., 319,96

Hüttig, C., \& Breuer, D. 2011, Phys. Earth Planet. Inter., 186, 111

Jackson, I., \& Rigden, S. M. 1996, Phys. Earth Planet. Inter., 96, 85

Karato, S.-I. 2008, Deformation of Earth Materials: An Introduction to the Rheology of Solid Earth (Cambridge University Press)

Karato, S.-I. 2011, Icarus, 212, 14

Karato, S.-I., \& Wu, P. 1993, Science, 260, 771

Katsura, T., Shatskiy, A., Manthilake, M. A. G. M., et al. 2009a, Phys. Earth Planet. Inter., 174, 86

Katsura, T., Yokoshi, S., Kawabe, K., et al. 2009b, Geophys. Res. Lett., 36, L01305

King, S. D., Lee, C., van Keken, P. E., et al. 2010, Geophys. J. Int., 180, 73

Léger, A., Grasset, O., Fegley, B., et al. 2011, Icarus, 213, 1

Leitzinger, M., Odert, P., Kulikov, Y. N., et al. 2011, Planet. Space Sci., 59, 1472

Lissauer, J. J., Fabrycky, D. C., Ford, E. B., et al. 2011, Nature, 470, 53

Martin, C. D., Crichton, W. A., Liu, H., et al. 2006, Geophys. Res. Lett., 33, L11305

Matyska, C., \& Yuen, D. A. 2006, Phys. Earth Planet. Inter., 173, 33

Mitrovica, J. X., \& Forte, A. M. 2004, Earth Planet. Sci. Lett., 225, 177

Oganov, A. R., \& Ono, S. 2004, Nature, 430, 445

Oganov, A. R., Brodholt, J. P., \& Price, G. D. 2001, Nature, 411, 934

Okada, T., Yagi, T., \& Nishio-Hamane, D. 2010, Phys. Chem. Miner., 38, 251

Ono, S., \& Oganov, A. R. 2005, Earth Planet. Sci. Lett., 236, 914

Pollack, H. N., Hurter, S. J., \& Johnson, J. R. 1993, Rev. Geophys., 31, 267
Pont, F., Aigrain, S., \& Zucker, S. 2010, MNRAS, 411, 1953

Ranalli, G. 2001, J. Geodyn., 32, 425

Romanowicz, B., \& Lambeck, K. 1977, Phys. Earth Planet. Inter., 15, 1

Rouan, D., Deeg, H. J., Demangeon, O., et al. 2011, ApJ, 741, L30

Sasaki, S., \& Nakazawa, K. 1986, J. Geophys. Res., 91, 9231

Seager, S., Kuchner, M., Hier-Majumder, C. A., \& Militzer, B. 2007, ApJ, 669, 1279

Senshu, H., Kuramoto, K., \& Matsui, T. 2002, J. Geophys. Res., 107, 5118

Shampine, L. F. 1994, Numerical solution of ordinary differential equations (Springer)

Sleep, N. H. 1990, J. Geophys. Res., 95, 6715

Sohl, F., Wagner, F. W., Hussmann, H., \& Grott, M. 2009, Terrestrial planets and satellites: Planetary Interiors, in Landolt-Börnstein: Numerical Data and Functional Relationships in Science and Technology, ed. J. E. Trümper (Springer)

Sotin, C., Grasset, O., \& Mocquet, A. 2007, Icarus, 191, 337

Stacey, F. D. 2005, Rep. Prog. Phys., 68, 341

Stacey, F. D., \& Davis, P. M. 2008, Physics of the Earth (Cambridge University Press)

Stamenković, V., Breuer, D., \& Spohn, T. 2011, Icarus, 216, 572

Stamenković, V., Noack, L., Breuer, D., \& Spohn, T. 2012, ApJ, 748, 41

Tachinami, C., Senshu, H., \& Ida, S. 2011, ApJ, 726, 70

Tosi, N., Yuen, D. A., \& Cadek, O. 2010, Earth and Planet. Sci. Lett., 298, 229

Tozer, D. C. 1972, Phys. Earth Planet. Int., 6, 182

Tsuchiya, T., Tsuchiya, J., Umemoto, K., \& Wentzcovitch, R. M. 2004, Geophys. Res. Lett., 31, L14603

Turcotte, D. L., \& Schubert, G. 2002, Geodynamics (Cambridge University Press)

Umemoto, K., \& Wentzcovitch, R. M. 2012, Earth Planet. Sci. Lett., 311, 225

Umemoto, K., Wentzcovitch, R. M., \& Allen, P. B. 2006a, Science, 311, 983

Umemoto, K., Wentzcovitch, R. M., Weidner, D. J., \& Parise, J. B. 2006b, Geophys. Res. Lett., 33, L15304

Valencia, D. 2011, Composition of Transiting and Transiting-only superEarths, in The Astrophysics of Planetary Systems: Formation, Structure, and Dynamical Evolution, ed. A. Sozzetti, M. G. Lattanzi, \& A. P. Boss (Cambridge University Press)

Valencia, D., O'Connell, R. J., \& Sasselov, D. D. 2006, Icarus, 181, 545 Valencia, D., O'Connell, R. J., \& Sasselov, D. D. 2009, Astrophys. \& Space Sci.,
322, 135

Valencia, D., Ikoma, M., Guillot, T., \& Nettelmann, N. 2010, A\&A, 516, A20

van den Berg, A. P., Yuen, D. A., Beebe, G. L., \& Christiansen, M. D. 2010, Phys. Earth Planet. Inter., 178, 136

van Hoolst, T., Sohl, F., Holin, I., et al. 2007, Space Sci. Rev., 132, 203

van Summeren, J., Conrad, C. P., \& Gaidos, E. 2011, ApJ, 736, L15

Wagner, F. W., Sohl, F., Hussmann, H., Grott, M., \& Rauer, H. 2011a, Icarus, 214, 366

Wagner, F. W., Sohl, F., Rückriemen, T., \& Rauer, H. 2011b, Physical State of the Deep Interior of the CoRoT-7b Exoplanet, in The Astrophysics of Planetary Systems: Formation, Structure, and Dynamical Evolution, ed. A. Sozzetti, M. G. Lattanzi, \& A. P. Boss (Cambridge University Press)

Winn, J. N. 2011, Transits and Occultations, in Exoplanets, ed. S. Seager (University of Arizona Press) 